
\title{
$\begin{array}{ll}\text { Research Square } & \begin{array}{l}\text { Preprints are preliminary reports that have not undergone peer review. } \\ \text { They should not be considered conclusive, used to inform clinical practice, } \\ \text { or referenced by the media as validated information. }\end{array}\end{array}$ \\ Fact checkers fail to overcome partisan divides in two of the world's largest democracies
}

\section{Rik Ray}

University of Illinois Urbana-Champaign

\section{Sakshi Bhalla}

University of Illinois Urbana Champaign

Harsh Taneja ( $\nabla$ harsh.taneja@gmail.com )

University of Illinois Urbana-Champaign https://orcid.org/0000-0002-4630-8911

\section{Article}

Keywords: fact-checkers, misinformation, ideological divisions, India, US

Posted Date: November 4th, 2021

DOI: https://doi.org/10.21203/rs.3.rs-1004012/v1

License: (1) This work is licensed under a Creative Commons Attribution 4.0 International License. Read Full License 
1 Fact checkers fail to overcome partisan divides in two of the world's largest democracies

2 Rik Ray, Sakshi Bhalla \& Harsh Taneja ${ }^{1}$

3 Institute of Communications Research, University of Illinois-Urbana Champaign

\begin{abstract}
Misinformation easily spreads on social media and fact-checkers have an important role in correcting falsehoods. Most misinformation is of a partisan nature and appeals selectively to users on the basis of ideology. Thus, it is possible that fact checks may not overcome existing ideological divisions on social media. We examine this separately for a slice of Twitter users, following certain partisan outlets from India and the US. In both cases, users of left-leaning news outlets are more likely to follow and share content by fact checkers. Followers of rightleaning outlets rarely follow or amplify fact checkers and only selectively engage to reply to posts by fact checkers. Our analysis of $7 \mathrm{mn}$ partisan news users from two of the world's largest democracies suggests that exposure to fact-checking therefore remains largely restricted to leftleaning Twitter users with little evidence that these interventions penetrate among right-leaning slices, where partisan misinformation also circulates.
\end{abstract}

Main

Social media, once hailed as harbingers of democratic change, are now seen as active sites for the propagation of political misinformation. The increased circulation of disinformation and misinformation potentially amplifies political polarization which has adverse consequences on democratic processes, public health, and can foment social unrest (Watts et al., 2021; Roozenbeek et al., 2020; Loomba et al., 2021; Narayanan et al., 2019). Social media facilitate news audiences to easily access alternative information sources, which could augment such effects, especially in the light of declining trust in mainstream media. Thus, strategies to identify and counter online misinformation have become necessary. Consequently, fact checkers organizations that specialize in identifying and correcting circulating pieces of misinformation are seen as playing an important role. Yet the extent and efficacy of fact checking initiatives in countering political misinformation, especially in overcoming partisan divides on social media remains questionable and understudied.

Our study foregrounds this question based on two considerations. First, social media platforms have exacerbated an ever-increasing supply of "news" sources, many of which are hyper-partisan in their political ideologies (Flaxman et al., 2016). A large proportion of these sources are not trusted news organizations, increasing their propensity to peddle partisan misinformation. Second, news consumption on social media is believed to be happening inside "echo chambers" or "filter bubbles" of people with like-minded political ideologies and attitudes (Guess et al. 2020).

Recent elections worldwide, most notably including the 2016 US presidential election, have centered scholarly focus on examining the role of social media in propagating political

\footnotetext{
${ }^{1}$ Corresponding author. Email: harsh.taneja@gmail.com
} 
misinformation exacerbating political opinion polarization. Although it is often difficult to trace the original source of partisan political misinformation, certain hyper-partisan "rumor entrepreneurs" use non-traditional news websites to actively produce and re-circulate old misinformation on social media platforms (Shin et al 2018). Exposure to partisan misinformation might reduce trust in mainstream news media (Ognyanova et al., 2020), which in turn, can lead people to turn to non-mainstream partisan news outlets (Fletcher \& Park, 2017). Further, personalization algorithms on social media could create "cybercascades" of misinformation and thus increase polarization (Sunstein, 2017). Partisan cues also motivate people to share political misinformation on social media platforms (Osmundsen et al., 2021; Bowyer \& Kahne, 2019). Likewise, ideological congruence is associated with partisan selective exposure and consumption of political misinformation (Guess et al. 2020). In sum, exposure to partisan outlets, especially on social media can further erode trust in mainstream news media as well (Guess et al., 2021), thus contributing to the possible formation of "echo chambers".

The evidence on whether online echo-chambers exist is somewhat mixed. While some studies point to social media acting as echo chambers that polarize public opinion (Dylko et al., 2017; Hong \& Kim, 2016), others have found evidence for depolarizing effects due to exposure to diverse opinions (Garrett et al., 2014; Barberá, 2014), and yet others have found no direct association between social media use and opinion polarization (Lee et al., 2018). Studies have found an indirect relationship between social media use and opinion polarization mediated by political news consumption (Choi \& Lee, 2015) and increased political engagement (Lee et al., 2018). Further, the mediating role of social media news use in polarizing political opinion is especially pronounced among individuals who frequently encounter like-minded information ( $\mathrm{Lu}$ et al., 2020). Thus, even though social media use may not directly facilitate the formation of political echo chambers, social media platforms, such as Twitter, that are widely used for sharing and consumption of news content, can deepen existing partisan divides, which contributes to increased polarization (Garimella \& Weber, 2017; Gruzd \& Roy, 2014).

The concept of independently verifying statements by politicians or published information in the public domain by nonpartisan groups or individuals have existed in media systems across the world prior to the Internet (Graves, 2018). However, the formalized practice of political fact checking can be traced to the emergence of US-based online fact checkers such as FactCheck.org, PolitiFact, and The Washington Post's Fact Checker in the mid-2000s (Amazeen, 2017). The subsequent growth of hyperpartisan news websites peddling misinformation and potentially enhancing political polarization has fueled a movement of fact checking initiatives across the globe (Mantzarlis, 2016), which are modeled on these outlets. However, the efficacy of such initiatives in reaching out to audiences who are most likely to be vulnerable to partisan misinformation is questionable (Guess et al., 2020). 
If the news diet of partisan social media users solely comprises like-minded information, as many fear, they are unlikely to encounter corrective fact checking information. But partisan news audiences may not be as insulated from attitude-dissonant information as they are likely to have more ideologically diverse social networks online than in real life (Lee et al., 2014). Thus, they may be unintentionally exposed to attitude-dissonant information on social media platforms. However, exposure to information which counters existing beliefs such as fact-checks of partisan misinformation may be completely ignored or even actively resisted by such individuals through rebuttals (Bail et al., 2018; Lu, 2019). While it is conceivable that partisan media audiences are more likely to engage with fact-checking content owing to a general distrust in media, such perceptions of media bias do not necessarily apply to news content from outlets that are selfselected by individuals (Barnidge et al., 2020). Thus, partisan news audiences may be prone to mistrusting fact-checking outlets which debunk partisan misinformation. Considering that a disproportionate amount of partisan misinformation promotes right-leaning perspectives (Guess et al., 2020), conservative social media news consumers may be less amenable to fact-checks. We thus posit that if partisan social media audiences indeed reside within filter bubbles, then corrections by fact checkers may not be equally likely to reach either side of the partisan divide. Yet large-scale empirical evidence of whether this is really the case remains elusive.

Social media is increasingly becoming the primary source of news content for individuals across the world, outpacing even news websites and apps in younger populations (Newman et al., 2021). Twitter is particularly popular among online news consumers, with $71 \%$ of American Twitter users obtaining news on the platform (Matsa \& Shearer, 2018). As such, considerable attention has been paid to the content dynamics of misinformation propagation on Twitter as well as strategies to counter them. Despite the growing popularity of fact-checking outlets on Twitter, there is at least some evidence suggesting that these outlets may not be as effective in dispelling partisan misinformation. Those who spread such misinformation on Twitter tend to do so via homophilous follower networks, whereas fact-checkers do not have as sizable communities (Shin et al., 2016). Further, fact-checking of political misinformation is less likely to be accepted by partisans (Margolin et al., 2018). Thus, our study aims to assess the reach of political fact checking initiatives among partisan news users on Twitter focusing on a partisan slice of news outlets based in the US, which has the highest share of users as well as in India, the world's largest democracy.

A majority of online news audiences are exposed to ideologically moderate news outlets. However, a small but vocal minority of partisans have disproportionately higher engagement with partisan news outlets (Guess, 2021), especially on Twitter (Shore et al., 2018). Accordingly, we analyzed the co-following patterns of partisan news outlets with fact checking outlets among Twitter users in these two countries. In the US, three news outlets each representing politically left and right partisan leanings were selected for the analysis. PolitiFact and Snopes were chosen as the two fact-checking outlets. In India, two English news outlets each representing left and 
right partisan leanings were selected alongside AltNews as the fact-checking outlet. (See methods for details on how we selected our sample of outlets and assigned them their ideological leaning).

The complete lists of followers for each of these outlets were collected in March 2021 to analyze the co-following patterns. In general, our results indicate that the following of partisan news outlets on Twitter is quite insular in both countries i.e., users are much more likely to cofollow news outlets which are ideologically aligned than not. Only a small percentage of followers of partisan outlets also follow fact-checking outlets. Interestingly, a larger share of followers of left leaning outlets follow fact checkers in both countries.

While co-following patterns can provide a general overview of the extent of fact checking outlets in reaching out to partisan news consumers on Twitter, motivations for following fact-checkers may vary depending on the user's ideological leanings. Shin and Thorson's (2017) analysis of fact-checking tweets during the 2012 US presidential election revealed that fact checking tweets were shared selectively by partisan users that portrayed their own candidate competently and the opposing candidate poorly. Their study found evidence of hostile media perception of fact checking outlets among both Democrats and Republicans, with Republicans being more concerned about the bias of fact checkers. On a similar note, an experimental study of the efficacy of political fact checking revealed that hostile media perception can be an unintended side-effect of such efforts (Li et al., 2021). Whereas retweets often indicate a user's trust and agreement with the content (Metaxas et al., 2015), replies can be used to express disagreement. Hence, we analyzed the co-following patterns of users who interacted with fact checking tweets through retweets and replies over a roughly 13-month period from February 2020 to March 2021 to identify the differences between followers of left and right leaning outlets. We find that followers of right leaning outlets in both the US and India are more likely to reply to fact checking tweets, while followers of left leaning outlets are more likely to retweet them.

\section{Results}

We first report the co-follower analysis for both US and India, which establishes whether fact checkers can reach across both sides of the partisan divide. Next we report findings of models which explain the likelihood of replying to and retweeting tweets posted by fact checkers.

\section{Co-following of partisan outlets and fact checkers}

As of March 2021, for the US focused sample of outlets, there were 5.43 million unique Twitter users who followed at least one of the six partisan news outlets, or the two fact-checking 
outlets. As Table 1 indicates, the news landscape is fragmented along ideological lines, with high co-following of outlets that lean either left or right, and a much lower incidence of cross-cutting following. As such, the co-following patterns within the left and right share certain similarities. A large proportion of followers of Daily Kos and Daily Wire, the smallest left and right leaning outlets respectively, also follow the larger outlets with similar partisan leanings. The crosscutting following patterns between the left and right leaning outlets are quite similar and considerably lower than within ideologically aligned outlets i.e., larger proportions of users cofollowed ideologically similar outlets than ideologically dissimilar outlets.

A larger proportion of followers of left leaning outlets also follow fact checking outlets than followers of right leaning outlets. On the other hand, a significantly higher percentage of followers of PolitiFact and Snopes exclusively follow left leaning outlets sampled in our study, $22.5 \%$ and $20.7 \%$ respectively, than right leaning outlets, $3.1 \%$ and $3.6 \%$ respectively. In contrast, a tiny fraction of the followers of fact checking outlets $(2.7 \%$ for PolitiFact and $2.3 \%$ for Snopes) follow both, at least one right and left leaning outlet. Overall, these findings indicate that the following of fact checking outlets within cross cutting or right leaning followers of partisan news media on Twitter remains extremely limited.

For the India focused outlets, there were 1.78 million unique users who followed at least one of the six outlets. Overall, the co-following patterns within these outlets are quite similar to what we observed for the US outlets. Even though the cross-cutting following on the basis of partisan leanings is quite low across the board, marginally higher percentages of users who followed right leaning outlets followed left leaning outlets than followers of left leaning outlets who also followed right leaning outlets.

Followers of the two left leaning outlets Scroll and The Wire have a larger tendency to follow either of the fact checkers compared to followers of the two right leaning outlets OpIndia and Swarajya. Conversely, followers of fact-checking outlets who also follow left-leaning outlets are a considerably higher proportion than those who follow right-leaning outlets. Notably, among followers of the most prominent fact checker (Altnews), we found that only $2.4 \%$ exclusively follow either of the two right leaning outlets, whereas half (49\%) exclusively follow either of the left leaning outlets. Only $8.4 \%$ of AltNews followers follow at least one left-leaning and one right-leaning outlet. This indicates a high degree of partisan insularity in the co-following patterns of partisan outlets and AltNews, with followers of AltNews more likely to follow leftleaning outlets and vice-versa.

Similarly, insular communities were detected from a hierarchical clustering using Jaccard distance and average linkage method to assess the similarity of these outlets based on cofollowing patterns (see Methods). Among the US-based outlets (Figure 1), a three cluster resolution groups the three right leaning outlets, left leaning outlets and fact checkers into their 
own clusters. At a two-cluster solution, the fact checkers merge with left leaning outlets to form one cluster, but the right leaning outlets remain their own cluster. Results from logistic regression models with the following of fact-checkers as outcomes also indicated that the following of right leaning outlets is comparatively less likely to be associated with the following of fact-checkers (Supplementary Tables 5-7).

We replicated the analysis for the six outlets based in India. The findings were largely similar to those for the US-based outlets. As demonstrated in Figure 2, the two right leaning outlets are the least dissimilar and clustered together, followed by the two left leaning outlets, which form their own separate cluster. Further, the left leaning cluster is more similar with AltNews, which then merges with BOOM. Hence, co-following patterns are the least similar between the right leaning outlets and the rest of the outlets. While the odds of following fact checkers are largely similar for the following of The Wire and Swarajya, following OpIndia is associated with considerably lower odds (Supplementary Tables 8-10).

\section{Replying to and retweeting fact-checkers}

Between February 4, 2020 and March 3, 2021, 147,494 unique users had retweeted original tweets posted by PolitiFact and Snopes 511,564 times. Further, 65,322 users had replied to these fact checkers, totaling 106,753 replies. Half of all users who either retweeted or replied to fact checkers were following at least one of the eight US-based outlets in our sample and they accounted for an even higher share of both retweets (78.9\%) and replies (63.2\%) posted by all users. During the same time period, 34,607 unique users retweeted original tweets posted by AltNews 120,214 times, and 14,704 unique users replied to them 21,362 times. 75\% of all such users who either retweeted or replied followed at least one of the six India-based outlets, accounting for $87.7 \%$ of all retweets and $78.6 \%$ of all replies. Thus in both countries a majority of engagement with posts by fact checkers is from users who follow these outlets.

Results from two separate penalized maximum likelihood logistic regression models with retweeting and replying to fact checking tweets as outcomes, and the following of the six partisan news outlets as predictors revealed that the interactions with fact checking content on Twitter are not uniform across the following of different partisan news outlets. Barring Slate (OR $=.55,95 \% \mathrm{CI}=[.54, .57])$, following left leaning outlets were associated with significantly higher odds of retweeting fact checks. Following right leaning outlets were associated with significantly lower odds of retweeting. Whereas following Newsmax was associated with a $51 \%$ reduction in odds of retweeting $(\mathrm{OR}=.49,95 \% \mathrm{CI}=[.47, .51])$, following Breitbart or Daily Wire were associated with even lower odds. 65,322 unique users replied to tweets from PolitiFact and Snopes among which 35,797 (54.8\%) followed at least one of the eight outlets. Interestingly, only following Slate was associated with a significant reduction in odds of replying $(\mathrm{OR}=.38,95 \% \mathrm{CI}=[.37, .39])$. Following Mother Jones, Breitbart, or Daily Wire were all 
associated with significantly higher odds of replying and it was more than double for those following Daily Kos or Newsmax.

Replicating the same logistic regression models on the Indian follower dataset also yielded similar results. Following either of the two left leaning outlets, The Wire, Scroll, or one of the fact checkers, AltNews, and BOOM were associated with significantly higher odds of retweeting fact-checking content than following Swarajya $(\mathrm{OR}=.67,95 \% \mathrm{CI}=[.63, .72])$ or OpIndia $(\mathrm{OR}=.51,95 \% \mathrm{CI}=[.48, .54])$. Among the 14,704 unique users who replied to the tweets, 11,076 users $(75.3 \%)$ followed at least one of the six outlets. Similar to what we observe in the US, the patterns of replying to fact-checking tweets by AltNews are quite different from those observed for co-following of the outlets and retweeting. Results from the logit model indicated that following right leaning outlets Swarajya $(\mathrm{OR}=1.58,95 \% \mathrm{CI}=[1.50,1.67])$ and OpIndia $(\mathrm{OR}=1.90,95 \% \mathrm{CI}=[1.80,2.00])$ were associated with much higher odds of replying to such tweets. While following Scroll was associated with marginally lower odds of replying to fact checking tweets, following The Wire was not found to be significantly associated with replying.

\section{Discussion}

For both Indian and US sets of outlets, we first find, as expected, significant evidence of selectivity in that news users follow either one of left or right leaning outlets, but not both. Twitter users in general who follow fact checking accounts are an even smaller group compared to the followers of these partisan accounts. More significantly, pertinent to our question, in both the US and Indian cases those who follow fact checkers are highly likely to follow accounts of news outlets that are perceived as left leaning, but not those on the right. Even if we consider the smaller following of these fact checking outlets as compared to a majority of the partisan outlets in our study, 20\% appears to be a ceiling for co-following fact checking and partisan news outlets. In summary, our findings suggest that exposure to fact checkers is both niche and largely restricted to the followers of left-leaning outlets. When we consider that at least a few of these user accounts may be bots, the task of fact-checkers to even penetrate these insular partisan bubbles appears more daunting, let alone successfully countering pieces of misinformation circulating within them.

Beyond co-following patterns, we examined two specific deeper forms of engagement on Twitter, replying and retweeting, which require more active user participation, implying that a user not only is exposed, but also affected by the message. Retweeting a post not only indicates a user's interest in the content but also their agreement and trust in the message (Metaxas, 2015). For outlets based in both the US and India, we find that consistent with their higher propensity to follow fact-checking handles, followers of left leaning outlets also are more likely to retweet their tweets. Only one left leaning outlet, Slate emerged as an exception, possibly due to its 
disproportionately large follower count, which increases the odds of the average follower being less likely to retweet in general. Regardless, the lower odds of retweeting among followers of right leaning outlets significantly hinders the reach and visibility of fact checking content within followers of right leaning users. Thus, a large proportion of users, followers of those who follow right leaning outlets and possibly more susceptible to misinformation, are unlikely to be incidentally exposed to fact checks.

Replies on Twitter, in sharp contrast to retweets, are more combative (Supplementary Material) and have been documented especially for interactions related to political topics both in the US and India. This explains our findings which indicate that users who follow right-leaning news outlets in both India and the US, although much less likely to retweet, are quite likely to reply to tweets by fact-checkers. For the US based outlets, we find that followers of all three right-leaning handles and the two smaller left leaning outlets, Mother Jones and Daily Kos were all more likely to reply to fact checkers. A plausible explanation is that users of niche hyperpartisan outlets are usually heavy news users, with disproportionately high political interest and therefore more likely to engage in online political discussions (Guess, 2021). Consistent with this reasoning, we did not observe this effect for Slate, as due to its relatively large following, its average follower is likely lower on political interest and hence less likely to reply. For Indian outlets, this higher propensity to reply is however exclusive to followers of right-leaning sites among those who are not following AltNews. It is conceivable that these are mainly "right wing trolls", paid handles who specifically attack fact checkers as the latter often correct right leaning partisan misinformation and hence are perceived to be aligned with the left (Campbell-Smith \& Bradshaw, 2019).

Although our findings suggest that online fact checking initiatives have limited following among partisan news audiences, they should be interpreted within the study setting and its associated limitations. Following a user account is an almost universal affordance across the major social media and content sharing platforms that these media outlets are active on, such as Twitter, Facebook, Instagram, and YouTube. While following a media outlet may be broadly indicative of a person's interest in the published content, it does not necessarily imply a meaningful engagement with the content. Nevertheless, the co-following pattern combined with the retweeting and replying patterns provide us with a broad, even if somewhat coarse approximation of online partisan news consumers' interest in fact checking content on Twitter.

Since information flows on Twitter are more asymmetrical due to its open network structure, further research is required to investigate whether our findings also hold for platforms with closed network structures such as Facebook (Kim \& Lee, 2016). Further, the primary audience motivations for using these platforms also vary. Affordances for interactions such as liking, retweeting/sharing, and replying can be considered more representative metrics of engagement on Twitter and Facebook, but some of these features are not natively supported on 
Instagram or YouTube. In the absence of easily accessible cross-platform engagement metrics, it is difficult to assess the complete reach of fact checking messages.

We did not formally investigate the content of the fact checking tweets or replies in detail. However, the limited cross-cutting following between followers of left and right leaning outlets and interaction with fact checking content suggest the failure of fact checkers to generate active interest among a significant majority of news consumers who might be susceptible to misinformation. Consequently, the efficacy of fact checking messages in dispelling misinformation and fake news becomes largely inconsequential. Despite the differences in volume of replies between followers of left and right leaning outlets in the US and India, we found that acceptance of fact checks is largely conditional upon users' following of the fact checking outlets. That is to say, a disproportionate number of followers of left leaning outlets who reply also follow fact checking outlets. Conversely, only a fraction of followers of right leaning outlets who reply follow fact checking outlets, and actively dispute the fact checks. Thus, even when fact checking messages make their way across to the right side of the partisan divide, they are resisted.

These patterns are largely consistent across the two countries in our study. Despite the limited scope, the similarities and differences in Twitter news usage between the two countries allows for some reasonable generalizations concerning fact checking initiatives. Approximately $13 \%$ of news consumers in the US and $19 \%$ of English news consumers in India use Twitter for receiving news content (Newman et al., 2021). These figures closely correspond to the global average of $13 \%$ of consumers who use Twitter weekly for consuming news (Newman et al., 2021) even though there exists a considerable gap in internet penetration between these two countries. Thus, we might expect similar results regarding fact checking in other countries with thriving digital native partisan news outlets as well. We deliberately restricted our sample of news outlets to digital native partisan news media since mainstream media outlets are less likely to publish misinformation based on partisan agendas on Twitter owing to more robust regulatory frameworks governing legacy media. Followers of mainstream media outlets and indeed, a vast majority of Twitter users are unlikely to exist in partisan echo chambers (Shore et al., 2018). Although we sampled only a handful of such partisan outlets, the results clearly indicate that fact checkers are struggling to reach across partisan divides on Twitter, after controlling for the follower counts. As such, followers of partisan outlets that are more likely to spread misinformation are even less likely to trust fact checkers and be exposed to such messages.

Overall, our analysis of two of the world's largest democracies reveals that fact checkers, at least on Twitter, have limited reach, which restricts their ability to cross the partisan divide. Specifically, even with their limited following, their followers are disproportionately more likely to follow left leaning outlets and it is a subsection of these left leaning followers who amplify fact checks. Those on the other side, i.e., followers of right leaning outlets only engage to reply 
(and purportedly counter) posts of fact checkers. Thus, exposure to fact checking content remains restricted among a clique of Twitter users who follow relatively niche left leaning outlets. We observe little evidence of it penetrating to the other side, where a lot of online partisan misinformation it hopes to alleviate circulates.

These results also corroborate previous findings that misinformation and false news spread further than factual news (Vosoughi et al, 2018), and audiences that are most likely to consume false news are also the least likely to seek out corrective information (Guess et al., 2020). A majority of news users consume their news from the more moderate and balanced outlets. However, those who follow niche partisan outlets are more politically motivated and likely to engage in selective sharing of partisan content, including one laden with misinformation, especially if it accords with their own ideology. Many would argue that political misinformation is more salient among extreme right leaning Twitter users. Thus, the inability of fact checking content to reach and circulate among the user communities on the political right is deeply concerning.

A few strategies that have been discussed to minimize the spread of misinformation include a greater scrutiny of political elites who publicly make false claims, minimizing the media coverage of such claims, and push fact checking messages more aggressively such that they become more difficult to avoid (Nyhan, 2021). Media outlets and social media platforms in particular, have to shoulder greater responsibility in minimizing the spread of misinformation through their channels. While it might be naïve to expect online hyperpartisan websites to revise their publishing approach, mainstream media outlets still count among the major sources of online news and as such, should minimize the coverage of false or misleading claims. Mainstream media outlets should also incorporate fact checking misleading claims by public figures as part of their core news production practices.

In addition to fact checking initiatives and identification of misinformation at the source, intervention strategies like inoculation and media literacy have also been found to be effective to varying degrees (Cook et al., 2017; Guess et al., 2020, Porter \& Wood,2021). But media literacy interventions may not have sustained effects, especially among motivated right leaning partisans in India (Badrinathan, 2020). There have also been suggestions to borrow some of the techniques typically used for spreading misinformation to instead spread corrective information (Shelby \& Ernst, 2013). This approach can be especially effective if such messages are promoted by the elites and opinion leaders within social networks. Thus, holding such elites accountable for the claims that they make and in turn, forcing them to publicly retract misleading information can go a long way in reducing the spread of misinformation (Nyhan, 2021).

Given that social media platforms act as news aggregators, mechanisms should be put in place to stem the flow of misinformation more proactively. In the wake of the COVID-19 
pandemic and the 2020 US presidential election, Twitter began tagging tweets which notified users of potentially misleading information. However, motivated partisan news consumers are unlikely to be convinced by such measures. While extreme partisans may remain unaffected, actively promoting tweets from and recommending fact checking accounts among followers of outlets known to peddle misinformation may help convince more moderate partisans. Thus, the identification of online spaces where misinformation is more likely to circulate or fact checks are not able to penetrate serves as a critical first step. Doing so would allow for more optimized targeting of fact checking messages. Needless to state, any meaningful intervention at combating misinformation would require collaborative efforts by social media companies, media outlets, policymakers, and academic researchers.

\section{Methods}

\section{Sampling Strategy}

Our study design was aimed at analyzing the behavior of users more likely to be selectively exposed to partisan misinformation. Based on prior research, most mainstream legacy media outlets cater to more moderate users, whereas more niche outlets have substantial user bases which lean more extreme on either side of the partisan divide. Thus, both for the US and India, we selectively sampled outlets which have explicit partisan leanings and a relatively niche follower base. That said, the political left and political right is not a universal classification that can be universally applied in each country. Thus in this section, we explain our choice of specific outlet for each country in more detail, also defining how we classify the outlets from either nation as right or left leaning.

US: Alongside partisan cable news channels, online media such as political blogs and partisan news websites started becoming increasingly relevant within the US political news landscape in the early 2000s. In 2020, the proportion of American adults who primarily received political news from online media was roughly equal to those who primarily tuned into television news (Pew Research, 2021). In many ways, the 2016 US presidential election catalyzed an explosive growth of hyperpartisan news outlets, in large part due to Donald Trump's continued attacks on legacy news media outlets, terming them as 'fake news.' Consequently, Breitbart News emerged as the cornerstone of an insular right-wing media ecosystem, with social media as a vital cog in the machinery (Benkler et al., 2017). Benkler et al.'s (2017) analysis of news engagement behavior on Twitter suggested that legacy media outlets such as The Washington Post, New York Times, CNN, and MSNBC were more popular among followers of Hillary Clinton than Trump followers. However, left leaning partisan outlets such as Huffington Post, Daily Kos, Mother Jones, were also popular among Clinton followers. In fact, two left leaning outlets, Occupy Democrats and The Other $98 \%$ had higher interaction rates on Facebook than right leaning outlets, suggesting that the popularity of hyperpartisan outlets are not restricted to conservative or right leaning news audiences. For our analysis, we selected three partisan outlets 
each to represent the right and left leaning slices of the US news ecosystem. While Slate, Mother Jones, and Daily Kos comprise the sample of left leaning outlets, Breitbart News, The Daily Wire, and Newsmax were selected to represent right leaning news sources. We deliberately excluded legacy media outlets such as CNN, New York Times, Fox News, etc. since their Twitter accounts are more likely to be followed by audiences situated on either side of the partisan divide.

The growing prominence of hyperpartisan outlets and the polarized political climate has also affected people's trust in the media. A majority of Americans believed that their primary news sources presented issues in a partisan manner and published information without complete verification during the 2020 election (Shearer, 2020). Further, Trump supporters were twice as likely as supporters of Joe Biden to be skeptical about fabricated information presented by their preferred news sources (Shearer, 2020). The COVID-19 pandemic and the accompanying politicization of the public health policy was a central news agenda in 2020 alongside the presidential election. As such, politically motivated misinformation pertaining to public health policies and voter fraud were rampantly promoted by a vocal minority of conservative Twitter users (Chen et al., 2021). Hence, fact checking initiatives became even more relevant within this landscape. We selected the two most prominent outlets dedicated to fact checking, PolitiFact and Snopes for our study.

India: The 2014 Indian general election has been called the country's "first social media election", characterized by intensive social media campaigning and extensive usage of social media analytics by the political parties (Khullar \& Haridasani, 2014). The right leaning Bharatiya Janata Party's (BJP) landslide mandate in 2014 was an inflection point in not just the socio-cultural landscape of India, but also within India's media ecosystem. Hindu nationalist populist discourses attacking the perceived liberal elitism of mainstream English news media (Bhat \& Chadha, 2020) and the encroachment on editorial independence at these outlets led to the establishment of several digital news platforms (Chaudhry, 2016). Notable among these are digital-native outlets such as OpIndia, Swarajya, The Wire, and Scroll, which have grown to become some of the most prominent news outlets in India.

OpIndia and Swarajya position themselves as platforms for right of center and right liberal ideas. Swarajya started as a weekly magazine in the 1960 s, founded by C. Rajagopalachari, an Indian statesman who was also known for classical liberal political views and floated his own political party as a conservative opposition to the socialist leanings of the Indian National Congress (Pillai, 1965). It was relaunched in 2014 as a digital-native news outlet, to act as "a big tent for liberal right of centre discourse that reaches out, engages and caters to the new India." OpIndia was founded in 2014 under the aegis of Swarajya but became an independent entity in 2018 and is the most popular right-wing online news portal in India (Bhat \& Chadha, 2020). The Wire was founded in 2015 following the resignation of Siddharth 
Varadarajan as the editor of The Hindu, as an independent news platform that has often criticized the BJP-government. Similarly, Scroll.in was founded by news professionals seeking greater editorial freedom in criticizing the Narendra Modi-led BJP government (Chaudhry, 2016). Given their overt political dispositions, demonstration of implicit or explicit bias in their reportage may well be construed inevitable. These news outlets have an active presence on Twitter and as such, studying the following patterns of users who follow these outlets can provide some indication of how online English news audiences in India consume partisan news.

Swarajya and OpIndia were selected to represent right-of-center partisan outlets, Scroll and The Wire represent left-of-center outlets, and AltNews and BOOM were the fact-checking outlets. Considering BOOM's comparatively smaller follower count on Twitter and its broader focus on "fact-driven journalism" as compared with AltNews' higher follower count and its explicit positioning as a "fact-checking website," we decided to pivot our subsequent analyses around the latter.

\section{Data Collection}

Combining the GET followers/ids and the GET users/lookup methods of the Twitter API v1.1, the followers list, and their fully populated metadata of each of these accounts were downloaded in March 2021. An initial cross-tabulation of the users was employed to analyze the proportion of co-following for each pair of outlets. We created two combined datasets of unique Twitter users who follow any one of the six outlets based in India $(n=1,783,776)$ or any of the eight outlets based in the US $(n=5,432,425)$ respectively, with the following of each outlet coded as dummy variables. Next, the followers of the partisan news outlets and the fact-checking outlets were grouped to investigate the cross-cutting nature of following outlets with opposing partisan leanings and fact-checking sites. Additionally, separate dummy variables were created for users who follow at least one left leaning or one right leaning outlet but do not follow any outlet with opposing ideological slant. However, some of these users may follow fact checking outlets. The average age on Twitter for users only following right-leaning outlets was slightly lower than the average of all users for both the US and Indian set of outlets (Supplementary Table 11, Supplementary Figure 3).

To analyze the retweeting and replying patterns, we first downloaded all the retweets and replies to original tweets posted by @AltNews, @PolitiFact, and @ Snopes from February 4, 2020 to March 3, 2021 using the full archive search endpoint of the Twitter API v2. Users who retweeted or replied to an original tweet were cross-referenced with the combined datasets for each country and were coded as separate dummy variables. The patterns of retweeting and replying among followers of the left-leaning, right-leaning, and fact-checking outlets were analyzed using cross-tabulation. Owing to the Twitter API's current limitations and policy 
519

restrictions, we were unable to compile a list of users who liked the original tweets as well as collect more advanced engagement metrics on the tweets.

\section{Cluster analysis}

To determine the similarity between partisan news outlets and fact checking outlets in each country, we conducted agglomerative hierarchical cluster analysis on both the US and India datasets. First, a similarity matrix was constructed for each dataset by calculating the Jaccard index for each pair of outlets. The Jaccard index for two outlets, $\mathrm{X}$ and $\mathrm{Y}$, is calculated as:

$$
\mathrm{S}_{\mathrm{Jaccard}}=\frac{a}{a+b+c}
$$

where, $a$ is the number of users following both outlets $\mathrm{X}$ and $\mathrm{Y}$,

$b$ is the number of users not following $\mathrm{X}$ but following $\mathrm{Y}$, and $c$ is the number of users following $\mathrm{X}$ but not following $\mathrm{Y}$.

The Jaccard similarity coefficient is preferred over other binary similarity measures since we are only interested in the co-following pattern between two outlets based on the total combined follower count of both outlets. For any given pair of outlets, not following either cannot be considered a reasonable measure of similarity in this particular context. The similarity matrix was then converted into a dissimilarity matrix by subtracting the Jaccard indices from 1 . The dissimilarity matrix was used to cluster the outlets using the average linkage method. In the average linkage procedure, the distance between two clusters $C_{1}$ and $C_{2}$ is calculated as the average of the distance between each point in $\mathrm{C}_{1}$ with every other point in $\mathrm{C}_{2}$. It is given as:

$$
d\left(C_{1}, C_{2}\right)=\frac{1}{n_{1} n_{2}} \sum_{u \in \mathrm{C}_{1}} \sum_{v \in \mathrm{C}_{2}} d(u, v)
$$

The robustness of the results was checked using various combinations of Dice dissimilarity coefficient alongside single and complete linkage methods on both datasets. The cluster dendrograms were almost identical in all the cases except while using the single linkage method on the US dataset. It produced chain-like clusters, a known limitation of single linkage clustering.

\section{Logistic regression}

Finally, to examine the association between the following of a given outlet on Twitter with the likelihood of retweeting and replying to tweets from fact checking outlets, we conducted a set of logistic regression analyses. We conducted two sets of analyses each for the outlets based 
555 in the US and India, with the replying to tweets and retweeting as the outcome variables on the 556 combined follower datasets. The predictors were a set of dummies for each news outlet 557 indicating whether a user followed a particular outlet. Due to the rarity of the events (retweeting 558 or replying) and to account for data with separation, Firth's penalized maximum likelihood 559 method (1993) was used to estimate the models using the brglm2 package in R (Kosmidis, 560 2017).

562 Data Availability

563

564 The data supporting the findings are available from the authors upon request.

565

566 Code Availability

567

568 The R code used for conducting the analyses are available from the authors upon request. 


\section{References}

Amazeen, M. A. (2020). Journalistic interventions: The structural factors affecting the global emergence of fact-checking. Journalism, 21(1), 95-111.

Bowyer, B., \& Kahne, J. (2019). Motivated circulation: How misinformation and ideological alignment influence the circulation of political content. International Journal of Communication, 13, 25.

Bail, C. A., Argyle, L. P., Brown, T. W., Bumpus, J. P., Chen, H., Hunzaker, M. F., ... \& Volfovsky, A. (2018). Exposure to opposing views on social media can increase political polarization. Proceedings of the National Academy of Sciences, 115(37), 9216-9221.

Badrinathan, S. (2020). Educative Interventions to Combat Misinformation: Evidence from a Field Experiment in India. American Political Science Review, 1-17.

Barberá, P. (2014). How social media reduces mass political polarization. Evidence from Germany, Spain, and the US. Job Market Paper, New York University, 46.

Barnidge, M., Gunther, A. C., Kim, J., Hong, Y., Perryman, M., Tay, S. K., \& Knisely, S. (2020). Politically motivated selective exposure and perceived media bias. Communication Research, 47(1), 82-103.

Barthel, M. (2021, August 3). 6 key takeaways about the state of the news media in 2020. Pew Research Center. https:/www.pewresearch.org/fact-tank/2021/07/27/6-key-takeawaysabout-the-state-of-the-news-media-in-2020/.

Benkler, Y., Faris, R., Roberts, H., \& Zuckerman, E. (2017). Study: Breitbart-led right-wing media ecosystem altered broader media agenda. Columbia Journalism Review, 3(2).

Bhat, P., \& Chadha, K. (2020). Anti-media populism: Expressions of media distrust by rightwing media in India. Journal of International and Intercultural Communication, 13(2), 166-182.

Campbell-Smith, U., \& Bradshaw, S. (2019). Global cyber troops country profile: India. Oxford Internet Institute, University of Oxford.

Chaudhry, L. (2016). Can the digital revolution save Indian journalism?. Columbia Journalism Review, 54(5), 80-93.

Chen, E., Deb, A., \& Ferrara, E. (2021). \# Election2020: the first public Twitter dataset on the 2020 US Presidential election. Journal of Computational Social Science, 1-18.

Choi, J., \& Lee, J. K. (2015). Investigating the effects of news sharing and political interest on social media network heterogeneity. Computers in Human Behavior, 44, 258-266.

Cook, J., Lewandowsky, S., \& Ecker, U. K. (2017). Neutralizing misinformation through inoculation: Exposing misleading argumentation techniques reduces their influence. PloS one, 12(5), e0175799.

Dylko, I., Dolgov, I., Hoffman, W., Eckhart, N., Molina, M., \& Aaziz, O. (2017). The dark side of technology: An experimental investigation of the influence of customizability technology on online political selective exposure. Computers in Human Behavior, 73, 181-190.

Firth, D. (1993). Bias reduction of maximum likelihood estimates. Biometrika, 80(1), 27-38.

Flaxman, S., Goel, S., \& Rao, J. M. (2016). Filter bubbles, echo chambers, and online news consumption. Public opinion quarterly, 80(S1), 298-320. 
612

613

614

615

616

617

618

619

620

621

622

623

624

625

626

627

628

629

630

631

632

633

634

635

636

637

638

639

640

641

642

643

644

645

646

647

648

649

650

651

652

653

654

Fletcher, R., \& Park, S. (2017). The impact of trust in the news media on online news consumption and participation. Digital journalism, 5(10), 1281-1299.

Garimella, V. R. K., \& Weber, I. (2017, May). A long-term analysis of polarization on Twitter. In Proceedings of the International AAAI Conference on Web and Social Media (Vol. 11, No. 1).

Garrett, R. K., Gvirsman, S. D., Johnson, B. K., Tsfati, Y., Neo, R., \& Dal, A. (2014). Implications of pro-and counterattitudinal information exposure for affective polarization. Human communication research, 40(3), 309-332.

Graves, L. (2018). Boundaries not drawn: Mapping the institutional roots of the global factchecking movement. Journalism Studies, 19(5), 613-631.

Gruzd, A., \& Roy, J. (2014). Investigating political polarization on Twitter: A Canadian perspective. Policy \& internet, 6(1), 28-45.

Guess, A. M. (2021). (Almost) Everything in Moderation: New Evidence on Americans' Online Media Diets. American Journal of Political Science.

Guess, A. M., Barberá, P., Munzert, S., \& Yang, J. (2021). The consequences of online partisan media. Proceedings of the National Academy of Sciences, 118(14).

Guess, A. M., Lerner, M., Lyons, B., Montgomery, J. M., Nyhan, B., Reifler, J., \& Sircar, N. (2020). A digital media literacy intervention increases discernment between mainstream and false news in the United States and India. Proceedings of the National Academy of Sciences, 117(27), 15536-15545.

Guess, A. M., Nyhan, B., \& Reifler, J. (2020). Exposure to untrustworthy websites in the 2016 US election. Nature Human Behaviour, 4(5), 472-480.

Hong, S., \& Kim, S. H. (2016). Political polarization on twitter: Implications for the use of social media in digital governments. Government Information Quarterly, 33(4), 777-782.

Khullar, A., \& Haridasani, A. (2014, April 10). India's first social media election. CNN. https://www.cnn.com/2014/04/09/world/asia/indias-first-social-mediaelection/index.html

Kim, C., \& Lee, J. K. (2016). Social media type matters: Investigating the relationship between motivation and online social network heterogeneity. Journal of Broadcasting \& Electronic Media, 60(4), 676-693.

Kosmidis, I. (2017). brglm2: Bias reduction in generalized linear models. $R$ package version 0.1, 5.

Lee, J. K., Choi, J., Kim, C., \& Kim, Y. (2014). Social media, network heterogeneity, and opinion polarization. Journal of communication, 64(4), 702-722.

Lee, C., Shin, J., \& Hong, A. (2018). Does social media use really make people politically polarized? Direct and indirect effects of social media use on political polarization in South Korea. Telematics and Informatics, 35(1), 245-254.

Li, J., Foley, J. M., Dumdum, O., \& Wagner, M. W. (2021). The Power of a Genre: Political News Presented as Fact-Checking Increases Accurate Belief Updating and Hostile Media Perceptions. Mass Communication and Society, 1-26.

Loomba, S., de Figueiredo, A., Piatek, S. J., de Graaf, K., \& Larson, H. J. (2021). Measuring the impact of COVID-19 vaccine misinformation on vaccination intent in the UK and USA. Nature Human Behaviour, 5(3), 337-348. 
Lu, Y., Ray, R., Ha, L., \& Chen, P. (2020). Social Media News Consumption and Opinion Polarization on China's Trade Practices: Evidence from a US National Survey. International Journal of Communication, 14, 18.

Mantzarlis, A. (2016, June 7). There's been an explosion of international fact-checkers, but they face big challenges. Poynter. https://www.poynter.org/fact-checking/2016/theres-beenan-explosion-of-international-fact-checkers-but-they-face-big-challenges/

Margolin, D. B., Hannak, A., \& Weber, I. (2018). Political fact-checking on Twitter: When do corrections have an effect?. Political Communication, 35(2), 196-219.

Matsa, K. E., \& Shearer, E. (2018). News use across social media platforms 2018. Pew Research Center.

Metaxas, P., Mustafaraj, E., Wong, K., Zeng, L., O'Keefe, M., \& Finn, S. (2015, April). What do retweets indicate? Results from user survey and meta-review of research. In Proceedings of the International AAAI Conference on Web and Social Media (Vol. 9, No. 1).

Narayanan, V., Kollanyi, B., Hajela, R., Barthwal, A., Marchal, N., \& Howard, P. N. (2019). News and information over Facebook and WhatsApp during the Indian election campaign. Data Memo.

Newman, N., Fletcher, R., Schulz, A., Andi, S., Robertson, C. T., \& Nielsen, R. K. (2021). Reuters Institute Digital News Report 2021. Reuters Institute for the Study of Journalism.

Nyhan, B. (2021). Why the backfire effect does not explain the durability of political misperceptions. Proceedings of the National Academy of Sciences, 118(15).

Ognyanova, Katherine, et al. "Misinformation in action: Fake news exposure is linked to lower trust in media, higher trust in government when your side is in power." Harvard Kennedy School Misinformation Review (2020).

Osmundsen, M., Bor, A., Vahlstrup, P. B., Bechmann, A., \& Petersen, M. B. (2021). Partisan polarization is the primary psychological motivation behind political fake news sharing on Twitter. American Political Science Review, 1-17.

Pew Research Center. (2021, February 2021). How Americans Navigated the News in 2020: A Tumultuous Year in Review [Report]. https://www.pewresearch.org/journalism/2021/02/22/how-americans-navigated-thenews-in-2020-a-tumultuous-year-in-review/

Pillai, J. R. (1965). " SWATANTRA"—EMERGENCE OF A CONSERVATIVE OPPOSITION PARTY. The Indian Journal of Political Science, 26(2), 21-33.

Porter, E., \& Wood, T. J. (2021). The global effectiveness of fact-checking: Evidence from simultaneous experiments in Argentina, Nigeria, South Africa, and the United Kingdom. Proceedings of the National Academy of Sciences, 118(37), e2104235118.

Roozenbeek, J., Schneider, C. R., Dryhurst, S., Kerr, J., Freeman, A. L., Recchia, G., ... \& Van Der Linden, S. (2020). Susceptibility to misinformation about COVID-19 around the world. Royal Society open science, 7(10), 201199.

Shearer, E. (2020). News use across social media platforms 2018. Pew Research Center. https://www.pewresearch.org/fact-tank/2020/11/02/two-thirds-of-u-s-adults-say-theyveseen-their-own-news-sources-report-facts-meant-to-favor-one-side/ 
696 Shelby, A., \& Ernst, K. (2013). Story and science: how providers and parents can utilize storytelling to combat anti-vaccine misinformation. Human vaccines \& immunotherapeutics, 9(8), 1795-1801.

Shin, J., Jian, L., Driscoll, K., \& Bar, F. (2017). Political rumoring on Twitter during the 2012 US presidential election: Rumor diffusion and correction. New Media \& Society, 19(8), 1214-1235

Shin, J., \& Thorson, K. (2017). Partisan selective sharing: The biased diffusion of fact-checking messages on social media. Journal of Communication, 67(2), 233-255.

Shore, J., Baek, J., \& Dellarocas, C. (2018). Twitter is not the echo chamber we think it is. MIT Sloan Management Review, 60(1), 1-5.

Sunstein, Cass R. \# republic. Princeton University Press, 2017.

Vosoughi, S., Roy, D., \& Aral, S. (2018). The spread of true and false news online. Science, 359(6380), 1146-1151.

Watts, D. J., Rothschild, D. M., \& Mobius, M. (2021). Measuring the news and its impact on democracy. Proceedings of the National Academy of Sciences, 118(15). 
711

712

713

714

Tables and Figures

Table 1. Vertical percentage of co-following among US outlets on Twitter and interactions with fact checking tweets.

\begin{tabular}{|c|c|c|c|c|c|c|c|c|c|c|c|c|c|c|}
\hline & Outlet (mil) & 1 & 2 & 3 & 4 & 5 & 6 & 7 & 8 & 9 & 10 & 11 & 12 & 13 \\
\hline 1 & Slate (1.802) & 100 & 36.6 & 40.2 & 4.6 & 2.6 & 2.3 & 16.8 & 14.8 & 73 & 0 & 15.2 & 12.1 & 24.1 \\
\hline 2 & $\begin{array}{l}\text { Mother Jones } \\
(.863)\end{array}$ & 17.6 & 100 & 47 & 2.8 & 1.5 & 1.5 & 15.5 & 14.6 & 34.5 & 0 & 14.1 & 18.6 & 35.5 \\
\hline 3 & Daily Kos (.292) & 6.5 & 15.9 & 100 & 1.3 & 0.7 & 0.8 & 8.3 & 6.5 & 11.4 & 0 & 7.1 & 10.6 & 20.3 \\
\hline 4 & $\begin{array}{l}\text { Breitbart News } \\
(\mathbf{1 . 4 8 5})\end{array}$ & 3.8 & 4.8 & 6.6 & 100 & 33.8 & 38.4 & 4.7 & 4.6 & 0 & 61.3 & 4.4 & 29 & 5.2 \\
\hline 5 & $\begin{array}{l}\text { The Daily Wire } \\
(.623)\end{array}$ & 0.9 & 1.1 & 1.4 & 14.2 & 100 & 16.4 & 1.5 & 1.8 & 0 & 26.5 & 1.5 & 15.6 & 2.2 \\
\hline 6 & Newsmax (.865) & 1.1 & 1.5 & 2.3 & 22.4 & 22.8 & 100 & 2 & 2.1 & 0 & 36.9 & 1.9 & 22.3 & 3.3 \\
\hline 7 & PolitiFact (.697) & 6.5 & 12.5 & 19.9 & 2.2 & 1.7 & 1.6 & 100 & 20.4 & 6.6 & 1 & 75 & 33.3 & 52.3 \\
\hline 8 & Snopes (.292) & 2.4 & 4.9 & 6.5 & 0.9 & 0.8 & 0.7 & 8.6 & 100 & 2.6 & 0.5 & 31.5 & 30.7 & 34.8 \\
\hline \multicolumn{15}{|c|}{ Percentage of followers who follow only left leaning or only right leaning outlets } \\
\hline 9 & $\begin{array}{l}\text { Left leaning only } \\
(2.359)\end{array}$ & 95.5 & 94.3 & 92.1 & 0 & 0 & 0 & 22.5 & 20.7 & 100 & 0 & 20.8 & 24.1 & 46.6 \\
\hline 10 & \begin{tabular}{|l} 
Right leaning \\
only $(2.275)$
\end{tabular} & 0 & 0 & 0 & 94 & 96.6 & 96.9 & 3.1 & 3.6 & 0 & 100 & 3.3 & 38.3 & 4.7 \\
\hline 11 & $\begin{array}{l}\text { Fact checkers } \\
(.930)\end{array}$ & 7.9 & 15.2 & 22.8 & 2.8 & 2.3 & 2.1 & 100 & 100 & 8.2 & 1.3 & 100 & 54.7 & 74.8 \\
\hline
\end{tabular}

Engagement with outlets ( $\%$ of followers who replied or retweeted either fact checking outlets)

\begin{tabular}{|l|l|c|c|c|c|c|c|c|c|c|c|c|c|c|c|c|}
\hline 12 & Replies & 0.2 & 0.8 & 1.3 & 0.7 & 0.9 & 0.9 & 1.7 & 3.8 & & 0.4 & 0.6 & 2.1 & & 100 & 12.6 \\
\cline { 1 - 10 } & Retweets & 1 & 3.2 & 5.4 & 0.3 & 0.3 & 0.3 & 5.8 & 9.2 & & 1.5 & 0.2 & 6.2 & & 27 & 100 \\
\hline
\end{tabular}

715

716

717

718

719

720

721

722

723

724
Note: For rows 1-11, the number of followers (in millions) for each outlet or group are indicated in parentheses. Rows 9 and 10 indicate groups of users who follow at least one right or left leaning outlet but do not follow any outlets with opposing ideological leanings. Some of these users may follow fact checking outlets. Row 11 indicates users who follow at least one fact checking outlet and may or may not follow partisan outlets. Rows 12 and 13 indicate users who replied and retweeted posts by fact checkers who followed at least one of the eight outlets. Figures in each cell from columns 1-13 represent the percentage of users who followed the outlet(s)/replied/retweeted (in column) who also followed the outlet(s)/replied/retweeted in the corresponding rows. 
Table 2. Vertical percentage of co-following among Indian outlets on Twitter and interactions with fact checking tweets.

\begin{tabular}{|c|c|c|c|c|c|c|c|c|c|c|c|}
\hline & Outlet (mil) & 1 & 2 & 3 & 4 & 5 & 6 & 7 & 8 & 9 & 10 \\
\hline 1 & \begin{tabular}{|l} 
The Wire \\
$(\mathbf{1 . 0 0 8 )}$
\end{tabular} & 100 & 70.1 & 14.5 & 19.1 & 55 & 58.9 & 89.3 & 0 & 53.3 & 71.3 \\
\hline 2 & Scroll.in (.398) & 27.7 & 100 & 8.3 & 12.9 & 30.6 & 43.4 & 33.5 & 0 & 28.1 & 36.3 \\
\hline 3 & OpIndia (.522) & 7.5 & 10.9 & 100 & 67.8 & 8.8 & 17.8 & 0 & 86.3 & 31.8 & 6.6 \\
\hline 4 & Swarajya (.272) & 5.2 & 8.8 & 35.4 & 100 & 6.3 & 14.8 & 0 & 42.2 & 20.6 & 4.6 \\
\hline 5 & AltNews (.326) & 17.8 & 25.1 & 5.5 & 7.5 & 100 & 52.9 & 15.6 & 1.5 & 63.3 & 82.3 \\
\hline 6 & BOOM (.065) & 3.8 & 7.1 & 2.2 & 3.6 & 10.6 & 100 & 3 & 0.8 & 14.7 & 17.3 \\
\hline \multicolumn{12}{|c|}{ Co-following based on combinations of outlets } \\
\hline 7 & \begin{tabular}{|l} 
Left leaning \\
only (1.027)
\end{tabular} & 90.9 & 86.3 & 0 & 0 & 49 & 46.8 & 100 & 0 & 46.3 & 67.6 \\
\hline 8 & $\begin{array}{l}\text { Right leaning } \\
\text { only (.509) }\end{array}$ & 0 & 0 & 84.1 & 78.7 & 2.4 & 6.2 & 0 & 100 & 25 & 1.9 \\
\hline \multicolumn{12}{|c|}{ Engagement with outlets (\% of followers who replied or retweeted Altnews) } \\
\hline 9 & Replies & 0.6 & 0.8 & 0.7 & 0.8 & 2.1 & 2.5 & 0.5 & 0.5 & 100 & 12.1 \\
\hline 10 & Retweets & 1.8 & 2.4 & 0.3 & 0.4 & 6.6 & 6.9 & 1.7 & 0.1 & 28.4 & 100 \\
\hline
\end{tabular}

728

729

730

731

732

733

734

735
Note: For rows 1-8, the number of followers (in millions) for each outlet or group are indicated in parentheses. Rows 7 and 8 indicate groups of users who follow at least one right or left leaning outlet but do not follow any outlets with opposing ideological leanings. Some of these users may follow fact checking outlets. Rows 9 and 10 indicate users who replied and retweeted posts by AltNews who followed at least one of the six outlets. Figures in each cell from columns 1-10 represent the percentage of users who followed the outlet(s)/replied/retweeted (in column) who also followed the outlet(s)/replied/retweeted in the corresponding rows. 
736 Table 3. Firth models testing the association between retweeting and replying to tweets from 737 PolitiFact or Snopes.

738

\begin{tabular}{|l|c|c|l|l|l|l|}
\hline Variables & & \multicolumn{2}{|c|}{ US Retweets } & & \multicolumn{2}{c|}{ US Replies } \\
\hline & & $\beta$ & S.E. & & $\beta$ & S.E. \\
\hline & & & & & & \\
\hline Slate & & $-0.59 * * *$ & 0.01 & & $-0.97 * * *$ & 0.02 \\
\hline Mother Jones & $0.77 * * *$ & 0.01 & & $0.37 * * *$ & 0.02 \\
\hline Daily Kos & $1.01 * * *$ & 0.01 & & $0.71 * * *$ & 0.02 \\
\hline Breitbart News & $-1.06 * * *$ & 0.02 & & $0.43 * * *$ & 0.01 \\
\hline The Daily Wire & $-0.82 * * *$ & 0.03 & & $0.63 * * *$ & 0.02 \\
\hline Newsmax & $-0.72 * * *$ & 0.02 & & $0.76 * * *$ & 0.01 \\
\hline PolitiFact & & $1.61 * * *$ & 0.01 & & $1.27 * * *$ & 0.01 \\
\hline Snopes & & $1.96 * * *$ & 0.01 & & $2.15 * * *$ & 0.01 \\
\hline Constant & & $-4.92 * * *$ & 0.01 & & $-5.89 * * *$ & 0.01 \\
\hline
\end{tabular}

740 Note: $\mathrm{N}=5,435,425$, *** $p<.001$, S.E. Standard Error. Extended model details are shown in 741 Supplementary Tables 1 and 2. 
742 Table 4. Firth models testing the association between retweeting and replying to tweets from 743 AltNews.

744

\begin{tabular}{|l|l|l|l|l|l|l|}
\hline & & \multicolumn{2}{|c|}{ India Retweets } & & \multicolumn{2}{c|}{ India Replies } \\
\hline & & $\beta$ & S.E. & & $\beta$ & S.E. \\
\hline The Wire & & & & & & \\
\hline Scroll.in & & $0.6 * * *$ & 0.02 & & 0.02 & 0.02 \\
\hline Swarajya & & $-0.4 * * *$ & 0.03 & & $0.46^{* * *}$ & 0.03 \\
\hline OpIndia & & $-0.67 * * *$ & 0.03 & & $0.64 * * *$ & 0.03 \\
\hline AltNews & & $2.92^{* * *}$ & 0.02 & & $2.23^{* * *}$ & 0.02 \\
\hline BOOM & & $0.76^{* * *}$ & 0.02 & & $0.71^{* * *}$ & 0.03 \\
\hline Constant & & $-6^{* * *}$ & 0.02 & & $-6.27 * * *$ & 0.02 \\
\hline
\end{tabular}

745

746 Note: $\mathrm{N}=1,783,776, * * * p<.001$, S.E. Standard Error. Extended model details are shown in 747 Supplementary Tables 3 and 4. 
$748 \quad$ Figure 1. US outlets clustering

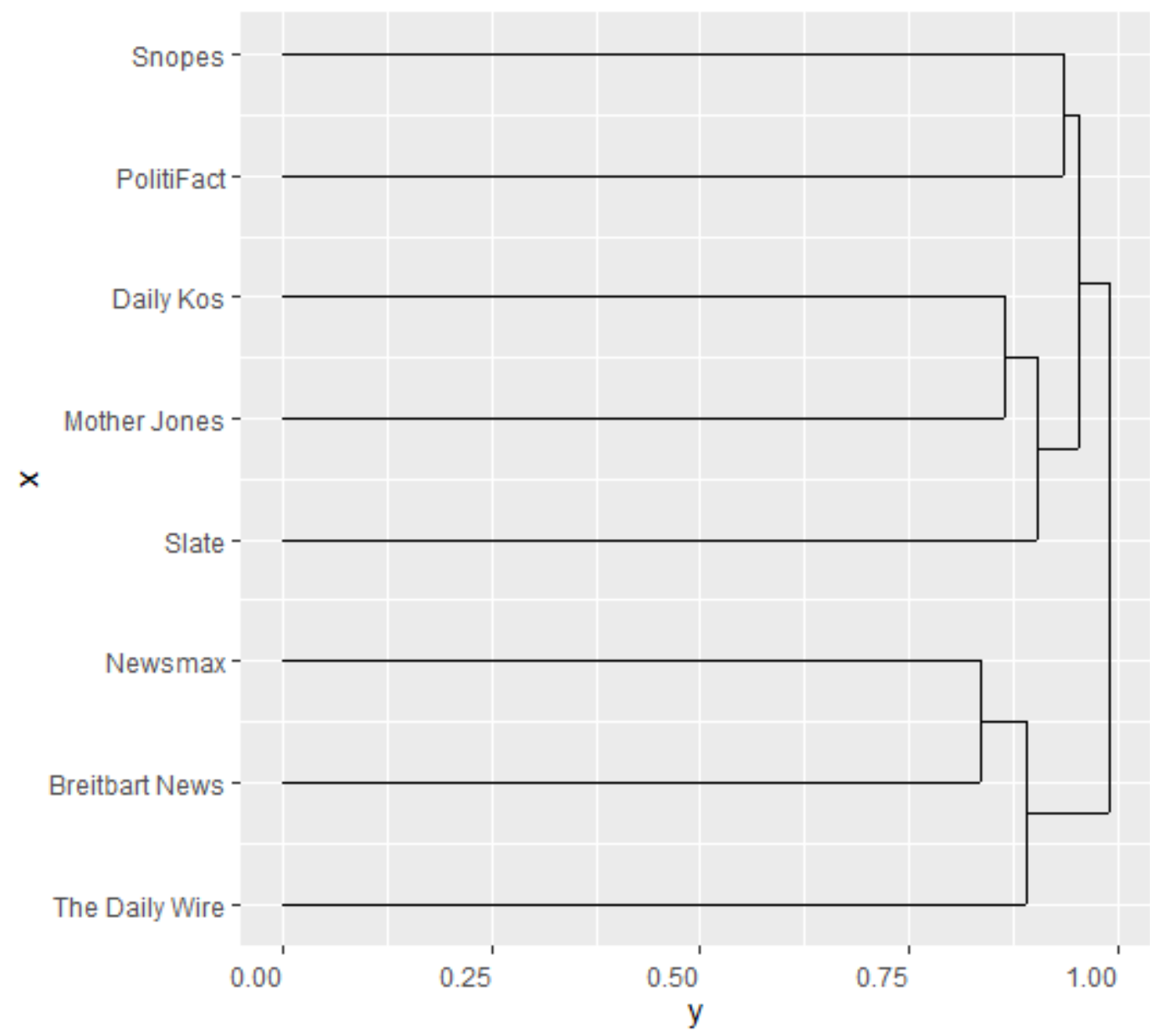

Dendrogram of the agglomerative hierarchical cluster analysis of the eight US-based outlets 751 using Jaccard distance measure and average linkage method. The outlets are arranged along the $752 \mathrm{x}$-axis with the Jaccard dissimilarity along the y-axis. 
753 Figure 2. Indian outlets clustering

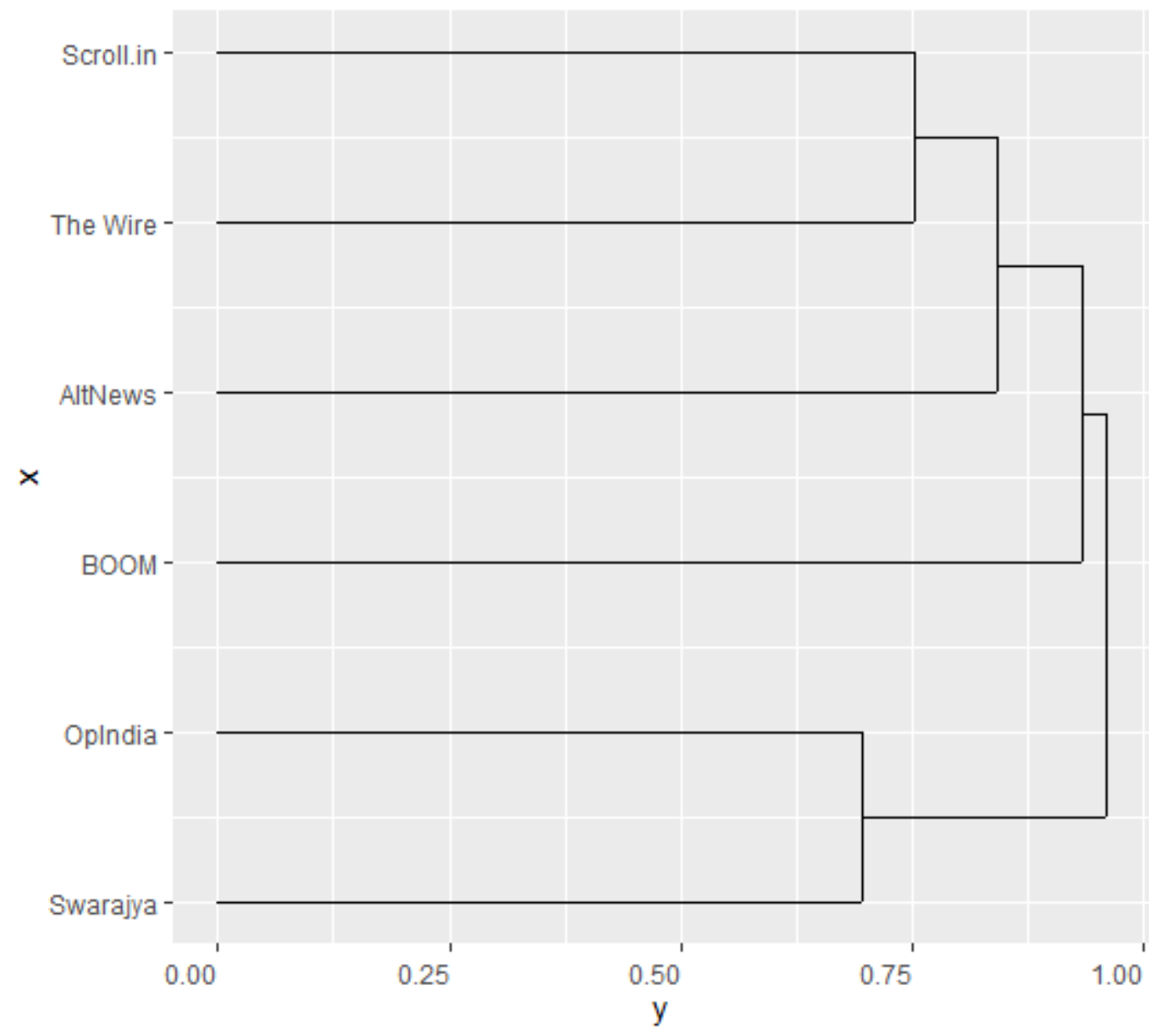

Dendrogram of the agglomerative hierarchical cluster analysis of the six India-based outlets using Jaccard distance measure and average linkage method. The outlets are arranged along the $757 \quad \mathrm{x}$-axis with the Jaccard dissimilarity along the y-axis. 


\section{Supplementary Files}

This is a list of supplementary files associated with this preprint. Click to download.

- Appendix.pdf

- nrreportingsummary.pdf

- nreditorialpolicychecklist.pdf 